\title{
INCREMENTO RÁPIDO DE LAS UNIVERSIDADES EN ESPAÑA UNA RED ESPACIAL DE CENTROS CON DIFÍCILES REORGANIZACIONES FUTURAS*
}

\author{
José M. ${ }^{a}$ Serrano MartíneZ ${ }^{1}$
}

\begin{abstract}
Resumen - Entre 1970 y 2000 se incrementa mucho el número de estudiantes universitarios en España. Sin embargo, a partir de 2001 comienza a descender, en especial los que siguen cursos en las Universidades estatales. La red de universidades ha crecido aún con mayor vigor durante esos años. Ha sido un proceso escasamente planificado. Ante la nueva realidad hay diferentes síntomas que aconsejan cambios estructurales en el sistema de funcionamiento de las Universidades españolas. Sin embargo, los intereses de las ciudades y de los gobiernos regionales constituyen una realidad de poder importante que dificulta cualquier proceso de reorganización territorial del sistema espacial de localización de las Universidades.
\end{abstract}

Palabras clave: Universidades, red de centros, sistema universitario español, organización territorial.

Resumo - INCREMENTO RÁPIDO DAS UNIVERSIDADES EM ESPANHA. UMA REDE ESPACIAL DE CENTROS COM DIFÍCIL REORGANIZAÇÃO FUTURA. Entre 1970 e 2000 aumentou muito o número de estudantes universitários em Espanha, passando de 156000 a 1581 000. Contudo, a partir de 2001 começou um processo de diminuição, em especial dos que frequentavam cursos em universidades estatais. Todavia, a rede de universidades aumentou de forma vincada durante esses anos. Tratou-se de um processo pouco planificado. No quadro da nova realidade detectam-se diferentes elementos que aconselham mudanças estruturais no sistema de funcionamento das universidades espanholas. Mas os interesses das cidades e dos governos regionais dificultam qualquer processo de reorganização territorial do sistema espacial de localização das Universidades.

Palavras-chave: Universidades, rede de centros, sistema universitário espanhol, organização territorial.

* Recebido: 4/05/2007. Revisto: 28/11/07. Aceite: 19/12/2007.

1 Catedrático de Análisis Geográfico Regional. Departamento de Geografía. Campus La Merced, Universidad de Murcia. 30001 Murcia, España. E-mail: jmserran@um.es 


\begin{abstract}
The SIGNIFICANT INCREASE IN THE NUMBER OF SPANISH UNIVERSITIES. A SPATIAL NETWORK FACED WITH FUTURE REORGANIZATION DIFFICULTIES. The number of university students in Spain underwent a very significant increase between 1970 and 2000, from 156000 to 1581000 . However, their number has been falling since 2001, particularly in the case of those attending public state universities. Nevertheless, the network of university institutions has kept expanding throughout, in a process that has not been adequately planned for. In view of the changing context, it is possible to identify a number of different symptoms that call for structural changes in the way in which the Spanish university system is organized. However, the vested interests of cities and regional governments introduce an important element of power that constrains any attempt to reorganize the spatial structure of the university system.
\end{abstract}

Key words: Universities, network Universities, Spanish University system, territorial management.

Résumé - LA MULTIPLICATION RAPIDE DES UNIVERSITÉS EN ESPAGNE. UN RESEAU DE CENTRES SPATIAUX QU'IL SERA DIFFICLE DE RÉORGANISER. De 1970 à 2000, le nombre des étudiants est passé de 156000 à 1581000 en Espagne, mais leur nombre a commencé à décroître à partir de 2001, surtout dans les universités publiques. Cependant le réseau des universités a continué à s'accroître sensiblement, selon un processus peu planifié. La nouvelle réalité rendrait souhaitable que des changements structurels soient introduits dans le système fonctionnel des universités espagnoles. Mais les intérêts des villes et des gouvernements régionaux rendent difficile toute réorganisation territoriale de ce système spatial de localisation.

Mots-clés: Universités, réseau de centres, système universitaire espagnol, organisation territoriale.

\title{
I. PLANTEAMIENTO
}

Dentro del conjunto de los servicios, los derivados de las actividades universitarias alcanzan una enorme significación. Basta pensar en las personas directamente afectadas: estudiantes, profesores, investigadores, personal de servicios, etc. En cualquier país suman cientos de miles, millones, los vinculados, de una u otra forma, con ellas. De ahí su destacada importancia en la economía urbana. Además, por su naturaleza intrínseca, de servicios avanzados, su impacto en la centralidad de las ciudades donde radican los centros se multiplica y acrecienta. Se puede llegar al extremo de que pequeñas ciudades se convierten en auténticos iconos urbanos mundiales gracias a la calidad y prestigio de sus instituciones universitarias.

La complejidad del tema permite enfoques plurales, desde diferentes disciplinas y de acuerdo con los objetivos concretos de análisis. Nuestras hipótesis centrales de trabajo son las siguientes:

- Durante un plazo breve, algo más de treinta años (1970-2007), en España ha aumentado mucho el número de estudiantes y el de Universidades. Tal ha sucedido al amparo de causas sociales y económicas favorables. 
Pero se ha carecido de un planeamiento suficiente. Ha ido actuándose sobre la marcha, al impulso de las necesidades.

- Los diferentes datos (a partir del 2000), señalan que estamos ante un cambio general de las circunstancias. La demanda estudiantil es ahora menor, de otra naturaleza, y las exigencias son distintas. Esto exige nuevos planteamientos; transformaciones, reacomodos; a causa de las propias deficiencias del sistema se aconseja su reorganización.

- Desde una perspectiva territorial todo apunta a que deberán producirse ciertos reajustes en la red de centros como consecuencia de las nuevas circunstancias nacionales y exteriores. Pero, dadas la realidad de la distribución del poder y las competencias existentes, todo ello se presenta cargado de enormes dificultades.

\section{AUMENTO ESPECTACULAR DE LOS ESTUDIANTES: UN PROCESO EXPANSIVO INTERRUMPIDO RECIENTEMENTE}

Durante tres décadas, 1970-2000, el número de estudiantes universitarios en España ha crecido con fuerza. Se ha multiplicado por diez, es decir, un mil por ciento. Su ritmo de ascenso ha sido mucho mayor al de la población, que en ese mismo tiempo apenas ha aumentado un veinte por ciento. Se trata de una evolución ascendente casi constante, si bien no del todo regular. Los datos del cuadro I, representados parcialmente en la figura 1 ayudan a comprobar tal proceso con mayor precisión.

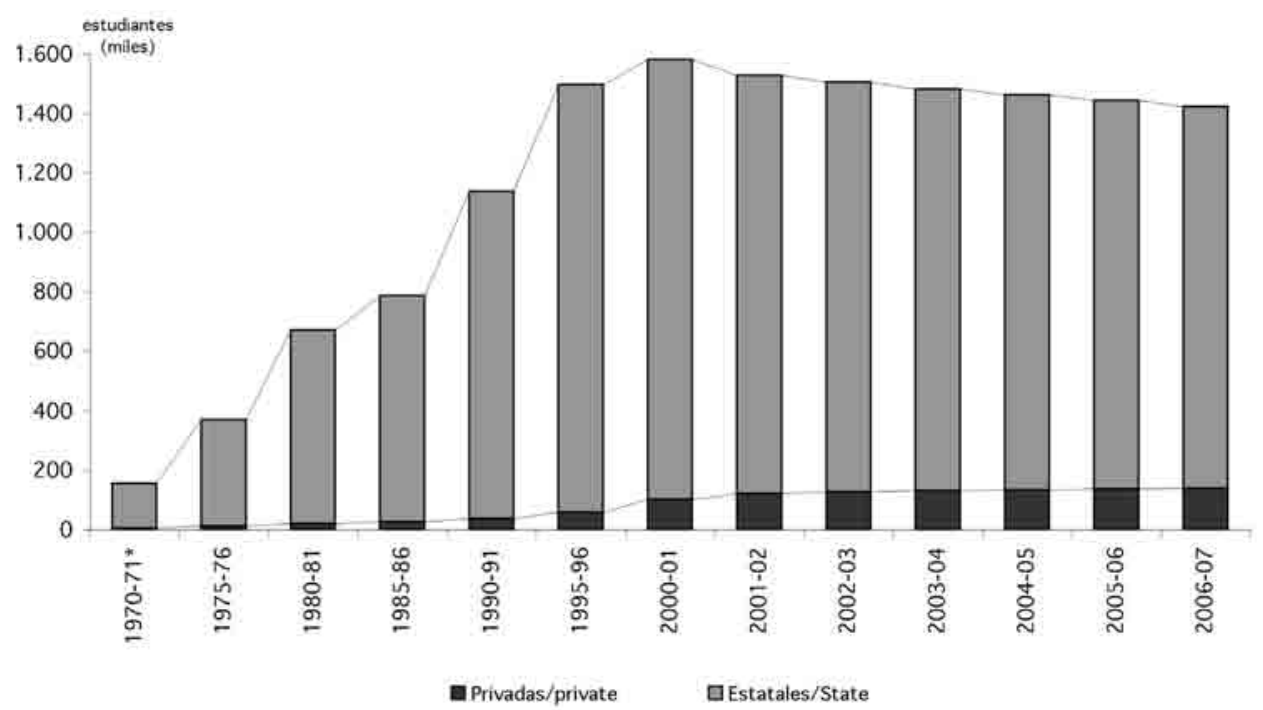

Fig. 1 - Estudiantes en centros estatales y privados (miles) (1970-2000 en lustros; detalle anual desde 2000).

Fig. 1 - Students attending state and private Universities (thousands) (quinquennial figures for 1970-2000; annual figures since 2000). 
Cuadro II - Evolución de los alumnos universitarios, centros estatales y privados.

Table I - The evolution of university students, in state and private enterprises.

\begin{tabular}{cccccc}
\hline Años & Estatales & \% & Privados & \% & Total \\
\hline $1970-71 *$ & 150094 & 96,09 & 6112 & 3,91 & 156206 \\
$1975-76$ & 358637 & 96,59 & 12669 & 3,41 & 371306 \\
$1980-81$ & 648637 & 96,84 & 21211 & 3,16 & 669848 \\
$1985-86$ & 759426 & 96,64 & 26454 & 3,36 & 785880 \\
$1990-91$ & 1099283 & 96,67 & 37945 & 3,33 & 1137228 \\
$1995-06$ & 1438875 & 96,07 & 58993 & 3,93 & 1497868 \\
$2000-01$ & 1479112 & 93,54 & 102303 & 6,46 & 1581425 \\
$2001-02$ & 1407369 & 92,03 & 121988 & 7,97 & 1529354 \\
$2002-03$ & 1378869 & 91,55 & 127379 & 8,45 & 1506248 \\
$2003-04$ & 1349248 & 91,04 & 132794 & 8,96 & 1482042 \\
$2004-05$ & 1328154 & 90,78 & 134743 & 9,22 & 1462897 \\
$2005-06$ & 1307691 & 90,57 & 136920 & 9,42 & 1443811 \\
$2006-07$ & 1283621 & 90,18 & 139975 & 9,83 & 1423396 \\
\hline
\end{tabular}

Fuente: Ministerio de Educación y Ciencia, www.mec.es

* No se incluyen los estudios en Escuelas Técnicas, superiores y medias, ni otros estudios superiores, como Bellas Artes, Música, que en esos años no se englobaban en los estudios universitarios; lo hacen a partir de esa fecha.

Es cierto que en 1970 se partía de una cifra reducida, en referencia a los promedios de la mayoría de los países vecinos de la Unión Europea (Bricall, 2000), con lo cual es más sencillo crecer con mayor fuerza. La cifra de estudiantes/población en 1970 era sólo 0,45 estudiantes universitarios por cada 100 habitantes. Por el contrario, en 2001, tal proporción asciende a 3,80. Este valor es de los más elevados de nuestro entorno comunitario (Eurostat, 2006).

No es sencillo precisar en pocas palabras las causas plurales, variadas, e incluso de diferente naturaleza, que han influido y contribuido a ese proceso de incremento de estudiantes tan significativo. Sólo es posible señalar de forma escueta algunas de las que participan con mayor fuerza.

- La mejora sustancial de la creación de riqueza en España significa un marco general donde es preciso situar esa evolución (Wright, 1977). La cual no ha cesado de evolucionar de manera favorable en años posteriores, a pesar de ciertos periodos de reajuste y crisis (Salmon, 1995).

- La transformación del sistema educativo, ha ampliando sus bases y extendido sus efectos a una población mucho mayor. De tal forma que casi toda la población en edad escolar ha podido cursar estudios medios, preparatorios para continuar su formación universitaria (Kagan, 1981).

- Dentro de ello, interesa destacar la ampliación de la política de becas y ayudas sociales para que las carencias económicas no constituyesen un 
impedimento a la hora de proseguir su acceso a la Universidad (Peach, 2001).

- Las elevadas tasas de natalidad de los años 50 al 70 del siglo XX significan generaciones numerosas de jóvenes que en esos años alcanzan la edad suficiente para acceder a su formación universitaria; dentro, todo ello, de un contexto poblacional de amplia y creciente representación urbana (Serrano y King, 1994).

- La propia fundación de nuevas universidades conlleva la ampliación sustancial de la red de centros, con una mayor densidad territorial. Eso representa menos dificultades para miles de jóvenes que desean acceder a una formación universitaria, al acortar las distancias desde sus domicilios a las nuevas Universidades (Guereña et al., 1991).

- Lo brevemente señalado se realiza dentro de un contexto socio-económico general expansivo, en el cual, el conseguir una titulación universitaria, significaba una cierta garantía inmediata de mejora en sus condiciones profesionales, elevación de salarios y ascenso en su situación social (Hernández, 1990).

Expuestas de manera muy resumida ciertas causas que explican el rápido incremento de los estudiantes universitarios en España, deseo añadir dos aspectos que considero de destacado interés.

Uno, hace referencia a la dualidad de estudiantes que siguen sus cursos en Universidades dependientes del sistema público (se denominan en general y para simplificar, Estatales). El otro, al resto de Universidades (vinculadas en su mayoría directa o indirectamente a instituciones relacionadas con la Iglesia Católica), aunque algunas se apartan de esa condición, todas ellas se nombran privadas. Se prefiere a utilizar para las primeras el término "estatales" al de "públicas", pues todas, unas y otras, están orientadas hacia un mismo servicio general, público. En el periodo analizado la parte de estudiantes que asisten a los centros estatales es abrumadora, en referencia a los que lo hacen a las Universidades privadas. No obstante, se advierte que, de forma lenta, pero continuada, se está produciendo un ligero aumento de los últimos frente a los primeros. Así, aunque en el último año de referencia la proporción alcanzada no llega siquiera el 10\%, interesa recordar que en sus inicios representaba menos del 4\%. Tal ascensión progresiva, tras un comportamiento inicial menos seguro, más tarde se afirma y prosigue sin interrupción. Ello resulta más llamativo a partir del año 2000, cuando dentro de un contexto general de retroceso de los estudiantes universitarios, continúa aumentando el de aquellos que siguen sus estudios en centros privados, frente a lo que acaece en los estatales. De ahí, pues, que las Universidades estatales y privadas evolucionan de forma diferente. En el fondo siguen lógicas dispares, dentro de estructuras respectivas que no son semejantes. Más adelante, al analizar la actual red de centros universitarios, se volverá con más detalle sobre estas cuestiones.

Otro aspecto, ya aludido, es que tras treinta años de aumento rápido del número de estudiantes, desde el curso 2000-01 cambia la tendencia, que prosi- 
gue hasta el presente. Cada año que pasa desde esa fecha desciende el total de alumnos inscritos en el conjunto de Universidades españolas. La mayor parte de ellas pierde alumnado (fig. 2).Ese comportamiento constante no es fruto de una casualidad ni de una coyuntura. Los retrocesos de los primeros años sugirieron, en principio, una situación excepcional. Pero cuando después se consolidan y se agrandan las cifras se toma conciencia de la nueva situación. Todo parece confirmar que se trata de un cambio estructural, motivado por causas profundas que es posible se mantengan y afiancen en lo sucesivo. Algunas reflexiones acerca de esto último se añaden a continuación; son de diferente naturaleza e importancia, pero al combinarse contribuyen a esos resultados finales apuntados:

- Una razón esencial es de carácter demográfico. Sucede algo similar a lo antes expuesto, pero en sentido contrario. El descenso acusado de la natalidad desde finales de los años setenta del siglo pasado, el cual se acentúa durante los ochenta, origina unas cohortes de edad menguantes, que son los que acceden cada año, a partir del 2000, en menor cuantía, a la Universidad. Como la natalidad casi permanece estancada desde los años noventa, en cifras muy reducidas (en torno al 9 por mil), no parece posible que se modifique al alza el número de posibles estudiantes atendiendo a este concepto. En años recientes, sin embargo, la población residente en España crece con fuerza; pasa de 40,8 a 45,1 millones de personas entre 2001 y 2007. Esto se debe, en especial, a la llegada masiva de inmigrantes (Serrano, 2006). Pero su condición social, económica, familiar y de edades, en general, no propicia su destacada incorporación inmediata, como estudiantes universitarios. Este panorama, pues, se mantendrá, al menos durante los próximos años, hasta que la inmigración se consolide, y los ahora niños y jóvenes que llegan, alcancen su edad de estudios universitarios, a la vez que, en el mejor de los casos, sus disponibilidades económicas también mejoren.

- Por otro lado tampoco debe olvidarse que la proporción de estudiantes universitarios alcanzada en España había crecido mucho, casi alcanzando un cierto exceso. Basta comparar que si en 1997 la proporción española entre 25 y 64 años que contaba con estudios universitarios era del 19\% (es decir un punto por debajo de la media de la OCDE), en 2004, esa proporción se situaba en el $26 \%$, (un punto por encima de dicho promedio). Tras su rápido incremento, la sobredotación lograda conviene tenerla en cuenta.

- A diferencia de lo que significaba en periodos anteriores, ahora superar muchos de los estudios universitarios, consiguiendo un diploma, no garantiza con igual seguridad ni cuantía una mejora económica. De ello se deriva, de alguna manera, su menor valoración social. Entre 2000 y 2006 los estudios realizados al respecto, revelan que los ascensos salariales mayores corresponden a quienes han logrado una especialización laboral, al margen de su formación universitaria (VV.AA. 2007). Es 
innegable que este cambio reduce el incentivo para seguir estudios universitarios a muchos. Ahondando en ese aspecto, deben aportarse dos cuestiones. Una, relativa a que la propia estructura general de la actividad productiva precisa de numerosos profesionales, de los diferentes campos de trabajo, formados al margen del mundo universitario (Mattelart, 2005). Dos, conviene preguntarse y reflexionar hasta qué punto los estudios y diplomas ofertados en la mayoría de las Universidades obedecen directamente a las necesidades reales demandas por el mercado. Se deduce que en muchos casos no es así. Sobre todo confirma esa aseveración comprobar el comportamiento dispar de los diferentes tipos de centros.

- Los datos de la figura 1, ya señalados, confirman un comportamiento diferenciado de los estudiantes inscritos en centros privados y estatales. Tomando como referencia las cifras más elevadas, las referidas al año 2000-01, con las del último contabilizado, 2006-7, los resultados difieren

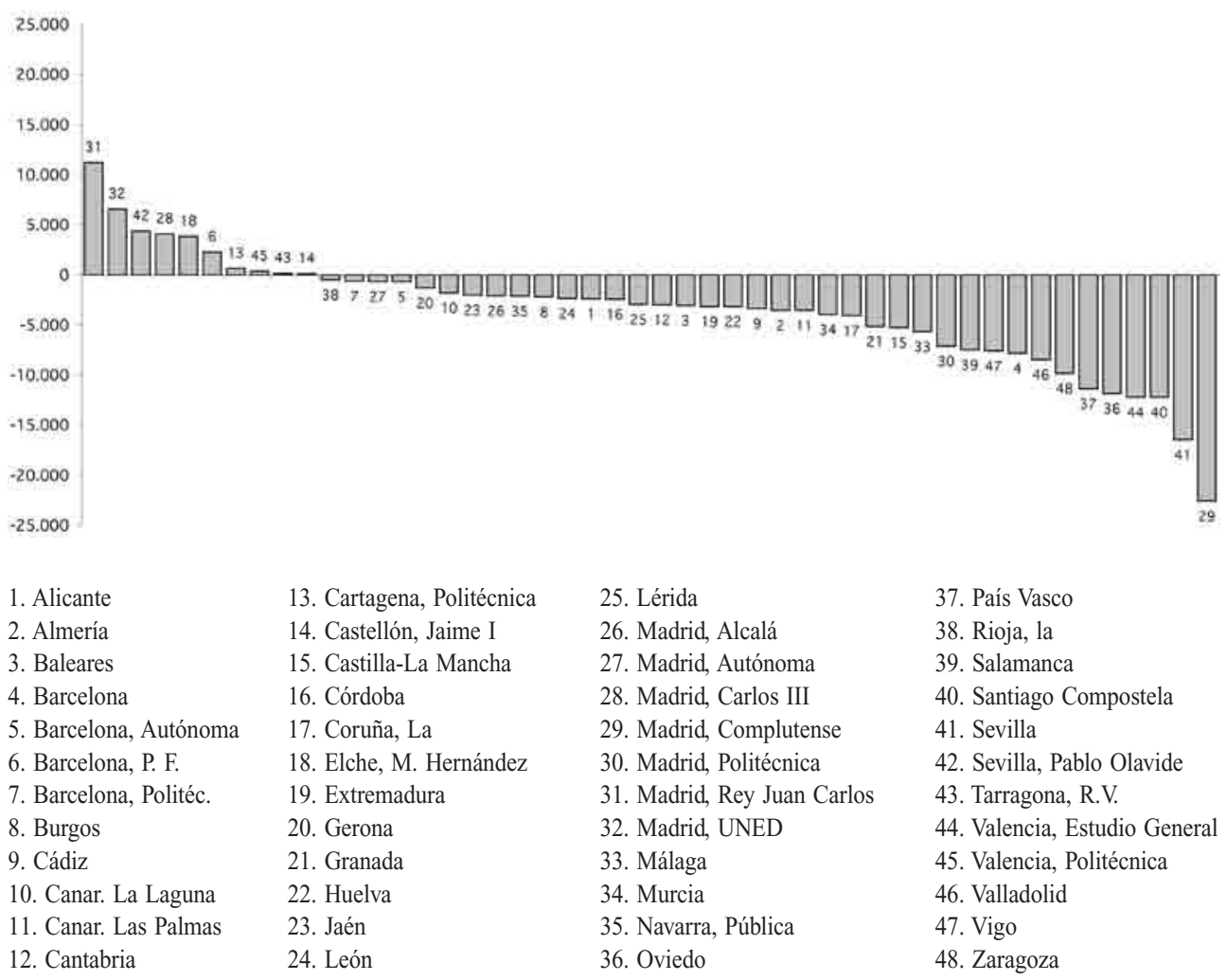

Fig. 2 - Universidades estatales, evolución del alumnado, 2000-2006. Fig. 2 - State universities, evolution in the number of students, 2000-2006. 
con claridad. Mientras que los centros estatales han perdido 195 491, los privados han aumentado en 37 672. Al comparar ambas cifras es más significativa la primera, frente a la segunda. Pero, sobre todo, interesa calibrar la dispar tendencia seguida por unos y otros.

- Interesa destacar que durante los últimos cursos la mayoría de las Universidades estatales han reducido su número de estudiantes (38 sobre 48). Incluso en varias de ellas las pérdidas han sido cuantiosas en datos absolutos y en valores porcentuales (fig. 2).

Lo analizado en este apartado muestra con claridad cuestiones bien diferentes. De un lado, que tras varios lustros de incremento rápido y continuado del número de estudiantes, se produce una inflexión con los comienzos del nuevo milenio. Por ahora no ha dejado de variar su tendencia regresiva. Todo parece anunciar que continuará varios años más. De otro, los resultados que muestran los centros estatales y los privados son claramente contrapuestos. Ello sugiere que siguen estrategias diferentes, de las que se deducen resultados y problemas de dispar naturaleza. Un análisis de la red de centros existente y de cuál ha sido su evolución reciente, puede ayudar a proporcionar ciertas claves que permitan conocer mejor la realidad a la que se enfrenta la Universidad en España.

\section{ACTUAL RED DE CENTROS UNIVERSITARIOS}

En 1970 la red de Universidades en España era escasa y poco densa. Había 15 universidades estatales y tres privadas. Estas tres últimas ligadas a la Iglesia y localizadas estratégicamente en Pamplona, Bilbao y Salamanca, cada una asociada a un sector de la iglesia católica, a saber: "opus dei", jesuitas y jerarquía eclesiástica regular. Otros centros docentes de dependencia religiosa, aunque participaban de alguna manera en la enseñanza superior, no habían alcanzado aún el status de Universidades. De esas tres, una era de muy reciente creación, Navarra, localizada en Pamplona y puesta en funcionamiento hacia la mitad del siglo pasado, en tanto que Deusto, en Bilbao, lo había sido en los comienzos del siglo XX. Debe recordarse que desde el siglo XIX, con los gobiernos de ideología liberal, se había mantenido por todos los ejecutivos una idea constante: la enseñanza superior debía estar en manos del Estado; siendo, pues, reticentes a cualquier participación eclesiástica (Álvarez Morales, 1972). La debilidad de estas Universidades privadas, dentro del sistema conjunto español, no era sólo por su número. También su volumen de estudiantes resultaba menguado, como ya se ha hecho referencia.

Las quince universidades estatales, constituían en 1970 la base esencial de la malla de enseñanza superior en España. Su red había crecido los años previos con la creación de las dos Universidades Autónomas (Madrid y Barcelona). Ello obedeció a dos causas esenciales: paliar la creciente masificación por la demanda en ascenso más intensa en las dos mayores ciudades 
españolas, y atender los deseos de autonomía universitaria. Su propia denominación enfatiza ese sentido (Miguel, 1973). Los restantes 13 centros casi coinciden con las regiones históricas tradicionales españolas que tienen sus orígenes en la Edad Media, con los antiguos reinos hispánicos, dentro de un claro proceso de territorialización regular (Grosetti y Losego, 1996). De hecho la mayoría de ellas remontan sus inicios fundacionales algunos siglos atrás, varias desde el siglo XIII al XVIII. Sólo ciertas excepciones confirman esa regla. La de Madrid (traslado a la capital española de la antigua y vecina Universidad de Alcalá, "Complutum"), y las tres fundadas en el siglo XX: La Laguna, Murcia y País Vasco. La malla territorial que formaban esos centros resultaba poco densa y algo descompensada. Laxa y débil porque amplios territorios españoles carecían de todo centro Universitario (Extremadura, Castilla-La Mancha). Descompensada, porque, al contrario, en otras áreas con menor densidad demográfica se mantenían más de un centro en servicio. Así y todo, desde una perspectiva territorial la escasa red de centros permitía atender a una reducida demanda de estudiantes. Todo ello, por supuesto, dentro de un modelo universitario muy limitado por los condicionantes políticos y sociales; la ausencia de libertad constituía una clara realidad (Montoro Romero, 1981). La prolongación temporal de tal situación había originado también una apreciable cerrazón nacional frente a otras comunidades científicas. La penuria económica de muchos años también era notoria por doquier en las instalaciones, equipamientos y servicios materiales (López Piñero, 1991). Pero, no debe olvidarse que esa Universidad, con todas las limitaciones apuntadas y otras que podrían añadirse, fue la que contribuyó a crear unos recursos humanos que hicieron capaz la enorme transformación y crecimiento económico logrados por España entre 1960 y 1980.

A partir de los años setenta el proceso de transformación del sistema universitario español fue rápido e intenso. Los centros estatales y privados siguen procesos disímiles, con lógicas dispares en la creación de nuevas universidades, tanto atendiendo a su evolución temporal, como a su localización territorial. Se añaden algunas precisiones al respecto a continuación, comenzando con los centros estatales:

En lo que se refiere al proceso de creación de nuevas Universidades estatales el ritmo de su evolución, fue muy vivo en los años posteriores a 1970. De los 15 centros contabilizados y en funcionamiento hasta ese año pasa en el curso 1980-81 a 29; diez años más tarde, el curso 1990-91, su total asciende a 35. En los cinco años siguientes su número continúa en aumento, así en el curso 199596, aún se produce la incorporación de otras 9 universidades. Sin embargo durante el segundo lustro de los noventa su cifra se acrecienta sólo con 4 centros más. Por consiguiente, vemos que la red de centros existente en 1970 (15 en total, la mayoría de las cuales tenían un origen lejano), al paso de sólo tres décadas, añade 33 nuevas universidades al incorporar 14 en la primera, 6 en la segunda y 13 en la tercera. Se triplica, pues, la base de la red inicial. A partir del 2000, hasta ahora, se ha detenido esa viva tendencia. 
Tras esos hechos es posible deducir una lógica en su disposición territorial. De un lado, se avanza hacia una realidad en la que cada región autónoma cuente al menos con una Universidad estatal. De otro, dentro de las regiones pluriprovinciales, todas las capitales de provincia de mayor tamaño demográfico, e incluso otros centros urbanos que no lo son, cuyo número de habitantes es elevado (cabe fijar su umbral medio en torno a los doscientos mil habitantes) presionan para que se ponga en servicio una Universidad sostenida con financiación estatal. También, en las principales aglomeraciones urbanas, donde las universidades tradicionales existentes estaban más saturadas de alumnos, se han creado nuevas universidades. El caso más destacado es Madrid; con menor fuerza, Barcelona, y, recientemente, Sevilla.

Por consiguiente, la lógica seguida que orienta todo ese proceso se basa en dos criterios esenciales: el de caminar hacia una distribución espacial más desparramada y atender el peso demográfico mayor de las áreas urbanas donde se ubican. Ambos aspectos son comunes en la distribución general de los servicios de rango elevado. Facilitar la accesibilidad de los usuarios (en este caso, estudiantes) y propiciar unos servicios de forma cómoda a una demanda potencialmente mayor. De esa manera puede decirse que apenas es posible encontrar en España áreas pobladas, que no tengan un centro universitario a menos de cien kilómetros de distancia (figura 3). Ello equivale a una isocrona en torno a una hora. Si se tratasen de otros servicios, comerciales, financieros, etc. la combinación de ambos criterios es razonable (Illeris, 1996). La cuestión clave se plantea en que una Universidad, más en la sociedad actual, no constituye un centro de servicios homologable a tales planteamientos tan sencillos y simples, similares a los de los bienes de rango elevado. El asunto es mucho más complejo y grave cuando se analizan otros aspectos de mayor importancia, referentes a la naturaleza, estructura y organización de los propios centros universitarios creados en España, como se aborda con más detalle en el epígrafe tercero de esta investigación. Para numerosos autores entre los muchos errores cometidos en el transcurso de los últimos años, tal vez el de más calado y significación, por las consecuencias negativas que conlleva a medio y largo plazo, ha sido el multiplicar las universidades, fraccionando unos recursos que habría que haber empleado, más bien, en mejorar las ya existentes. Pues el criterio que debe aplicarse a la Universidad es más el de calidad que el de cantidad. A nadie escapa que las muy costosas infraestructuras que necesita una Universidad, merecedora de ese nombre en propiedad, hacen por completo irracional que se las pretenda distribuirlas por doquier como si fuesen gasolineras, con el pretexto de satisfacer la falsa necesidad juvenil de estudiar sin moverse de casa.

Por su parte, las Universidades privadas han seguido en su expansión y puesta en funcionamiento otro ritmo y estrategias diferentes. Así, por ejemplo, cabe añadir lo siguiente: su reducido número de tres, umbral del que se parte en 1970, muestra en los primeros años un aumento pausado, una sola creación esa década y ninguna en la siguiente. Se llega, pues, a 1991 con 


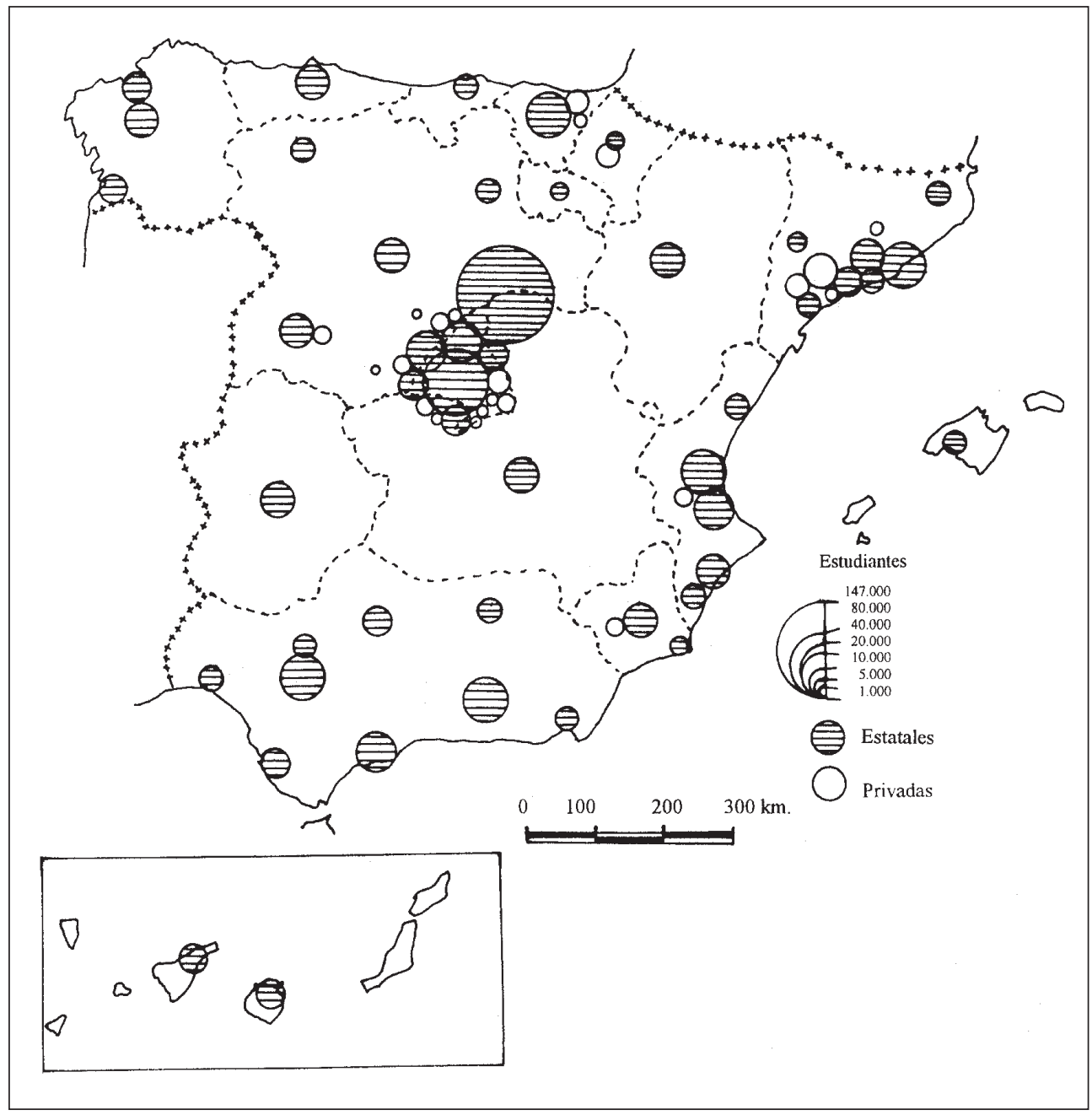

Fig. 3 - Red de Universidades en España, número de estudiantes en 2007

Fig. 3 - Spanish Universities network, number of students in 2007

sólo cuatro centros, todos vinculados a las instituciones religiosas, católicas, ubicados en Madrid, Salamanca, Navarra y Bilbao. Por el contrario, en los diez años que transcurren entre 1991-2001, se ponen en funcionamiento diez nuevas universidades. Más del doble de las previas existentes. Ese vigor se mantiene en el lustro posterior, incorporando otras nuevas ocho universidades, alcanzando así la cifra actual de 22 centros. La oferta universitaria privada, pues, ha sido más tardía que la estatal, aunque no menos dinámica. En datos absolutos, durante los 35 años analizados incorpora 19 centros frente a los 33 estatales. Pero en valores proporcionales su ascenso ha sido más fuerte, 
la base inicial la ha multiplicado por siete, frente a la estatal que lo ha hecho por tres.

El mayor esfuerzo en la creación de centros, en su primera fase, (años noventa), coincide con un vivo ascenso en la demanda de puestos escolares, con un masivo aumento de nuevos estudiantes. Pero, sucede lo contrario, en su última fase, primer lustro del nuevo milenio, en que coincide una reducción del número total de estudiantes universitarios frente a una oferta creciente de nuevas Universidades privadas. Tal combinación, en apariencia, parece un contrasentido. No lo es en la realidad, pues la suma global de estudiantes que siguen sus cursos en universidades privadas, no ha cesado de incrementarse en datos absolutos y proporcionales. Su cuota de mercado ha registrado una evolución favorable siempre, hasta el presente. Esto último se explica, en parte, al calibrar su estrategia territorial de localización de centros. Hasta ahora se caracteriza por los siguientes aspectos (se aconseja analizar la figura 3 , señalada):

- En la ciudad de Madrid, el entorno de su aglomeración metropolitana o bien en ciudades próximas a ella, se concentra su mayor número: un total de trece centros.

- El segundo ámbito metropolitano español y sus aledaños, Barcelona, suma cuatro universidades.

- Las cinco restantes se dispersan en otras tantas ciudades ubicadas en puntos distantes del conjunto nacional: Pamplona, Bilbao, Salamanca, Valencia y Murcia. Interesa matizar que la organización más flexible de estas universidades permite extender su actividad, parcial y sectorial a otras ciudades. Pero, ello no es óbice para deducir cuáles son las líneas básicas que orientan su estrategia de localización espacial. Parece evidente que no han pretendido dispersar sus centros, constituyendo una red territorial que abarque el conjunto de España. Por el contrario, su estrategia básica se ha dirigido a concentrar su oferta en las principales áreas metropolitanas, en especial en torno a la que es el núcleo esencial del sistema urbano español, Madrid. Ahí se aglutina más de la mitad de los centros. Tal estrategia es comprensible si se reflexiona sobre su lógica de fondo. Por su propia naturaleza las Universidades privadas tienen que autofinanciarse. Acogiéndose a las ventajas que ofrece el comportamiento del mercado encuentran sus mejores beneficios. Las condiciones básicas que buscan radican en: la mayor concentración de población, el nivel económico más elevado y una accesibilidad fácil, que permite ampliar con comodidad su mercado potencial. Además, los efectos de sinergia también cuentan. Otras condiciones de naturaleza política regional, tampoco deben olvidarse, dadas las competencias ejercidas por estos gobiernos, a la hora de la pertinente autorización inicial $\mathrm{y}$, después, de las titulaciones a ofrecer. 


\section{IV. ¿CRISIS DE UN MODELO TERRITORIAL UNIVERSITARIO? ¿HACIA QUÉ REESTRUCTURACIÓN? SUS IMPLICACIONES ESPACIALES}

El planteamiento y la comprensión de todas las actividades y tareas actuales de la humanidad deben hacerse dentro del escenario de un mundo globalizado. La sociedad mundial cada vez funciona más como una red integrada de relaciones de dispar naturaleza (Castells, 2005). Todos los aspectos relacionados con la sociedad del conocimiento, la investigación y la innovación alcanzan, en ese sentido, una prioridad mayor aún. Los conceptos intrínsecos propios de la Universidad, que comienzan a desarrollarse de forma palpable hace varios siglos, innovadores en su tiempo, adquieren ahora un sentido más pleno aún, en concordancia con su propia denominación. Conscientes de esa realidad ineludible, en un marco territorial más reducido, la Unión Europea, desde hace varios años trabaja por imponer una coordinación en este campo, plasmado recientemente en el denominado Espacio Europeo de Educación Superior (OECD, 1998). Los horizontes abiertos con el denominado marco de Bolonia ofrecen una esperanza. Se advierte con claridad que es preciso superar del todo los planteamientos regionales y nacionales, limitados y singulares, que dificultan una movilidad fácil e intensa de los sujetos implicados (estudiantes y profesores); se precisa crear escenarios que favorezcan una colaboración fecunda. Sólo mediante ese proceder se podrá hacer frente a una competitividad cada vez más intensa y agresiva (Almarcha, 2001).

Ese contexto general debe aplicarse de lleno a cualquier política universitaria nacional, en este caso, española; sean cuales fueren los planteamientos concretos a dilucidar, de naturaleza estructural, también conllevan una visión territorial. Igualmente, por supuesto, afectan directa e indistintamente a los centros gestionados por el Estado, los gobiernos regionales o por instituciones privadas, relacionadas con las instituciones religiosas o con entidades civiles.

Al centrarnos en el análisis de la realidad española actual (pero que encuentra escenarios parecidos o similares en otros países europeos), parece evidente (se ha aludido en varias ocasiones) que las circunstancias que rodean a los centros privados son diferentes a los que caracterizan a la mayoría, el sistema denominado estatal. Por consiguiente, cualquier estudio que aborde su comportamiento, posibles reestructuraciones y sus consecuencias territoriales, deberá realizarse de forma separada. Lo cual no es inconveniente para que, al final, deban relacionarse, ensamblándose ambas redes, dado que las dos conviven en plena relación, sirven al mismo mercado, si bien le hacen frente de diferente forma, y confluyen en similares objetivos finales para la sociedad. Dado lo complejo del asunto, y las limitaciones de espacio, se expondrán al respecto una serie de reflexiones de manera resumida y esquemática, circunscribiendo su análisis a la red de centros más numerosas y extensa, las universidades vinculadas al Estado y demás gobiernos regionales de las Comunidades Autónomas. 
- La reducción progresiva del total de estudiantes y su descenso casi general en la mayoría de las universidades aconseja no crear nuevos centros. Carece de sentido aumentar una oferta de plazas que se comprueba excesiva en la mayoría de las titulaciones. La red espacial de centros existente parece que, a corto plazo, se mantendrá en su actual dimensión, salvo ligeros cambios en una u otra dirección.

- La reducción de alumnos, y en general la menor presión de otros elementos de la vida universitaria, quizás constituye una ocasión para dedicar los esfuerzos de inversión en incrementar la calidad de la oferta de todos los servicios. Pasada la época de un aumento constante de estudiantes, cuya atención era la realidad más apremiante, ahora los problemas son otros.

- La creciente competitividad que introducen las universidades privadas puede y debe constituir también un aliciente para impulsar los servicios ofrecidos. Es necesario adaptarse al mercado general español, sin perder de vista la escala mayor que ofrece el espacio Comunitario Europeo y un mundo abierto y globalizado. Hace falta una adecuación del sistema educativo a la realidad presente, al mercado de trabajo y a las necesidades de la economía. Si bien, tampoco hay que seguir estrictamente la demanda del mercado laboral porque se acabaría creando especialistas de actividades que en diez años podrían dejar de existir.

Estas reflexiones generales parece fácil aceptarlas por cualquiera. Lo que resulta mucho más complejo es fijar los objetivos concretos a seguir para realizar esas transformaciones (Lamo de Espinosa, 2001). Aunque constituye un asunto complejo, cada vez se destaca con más intensidad el protagonismo creciente que aportan las Universidades norteamericanas. Atendiendo a la mayoría de los resultados su modelo destaca por la superioridad que encierra y aporta (Laszlo, 1996). Si bien es arriesgado sintetizar las razones de su éxito, se suele convenir que están ligadas, en esencia, a los siguientes aspectos:

- Flexibilidad institucional, gestión no burocrática de la investigación y la enseñanza.

- Significación destacada de los estudios de doctorado, con la consiguiente integración de estudiantes en la investigación.

- Enorme valor dado a la investigación, haciendo del doctorado, como punto inicial, un eslabón esencial, buscando siempre la excelencia académica.

Si se aceptan tales elementos, como presupuestos de partida, todo lo que constituya una aproximación a los mismos será favorable, y viceversa. Sin embargo, cuando se analiza la realidad de nuestro sistema universitario hay que reconocer que se encuentra muy alejado de tales presupuestos (Embid Irujo, et al., 2001). Pues, más bien, se caracteriza por: 
- Un funcionamiento claramente marcado por el predominio de la burocracia. Además, un sistema normativo en el que se superponen y, con frecuencia, se interfieren, tres niveles de actuación. De una parte, el gobierno autónomo, propio de cada universidad se orienta hacia una gestión que controla el funcionamiento cotidiano de los centros, dejando una discrecionalidad reducida a las unidades de investigación y docencia, los Departamentos. De otra, los respectivos gobiernos regionales, de quienes dependen directamente la gestión y la financiación universitaria, también representan una clara barrera que recorta la flexibilidad de funcionamiento de las universidades, estableciendo pautas de organización dominadas por los reglamentos y las normativas de control. Por último, el gobierno central, a través del ministerio correspondiente, lleva a cabo, así mismo, una clara tarea de coordinación, lógica, que persigue la homogeneización nacional necesaria. Lo peculiar es que eso se traduce fundamentalmente en la búsqueda a ultranza de una uniformidad lo más estricta posible de todas (Cabrera Montoya, et al., 2004). Parece que se ha pasado del tradicional centralismo español a un recelo hacia las tendencias centrífugas de ciertas regiones; lo cual, dada la realidad de ciertos casos, añade alguna comprensión (Romero, 2006). Sin duda, semejantes cuestiones son afrontadas y resueltas con mayor efectividad y agilidad por las universidades privadas. Entre otros aspectos, porque la organización de sus recursos humanos (elemento de gran importancia), no se fundamenta en el sistema funcionarial, sino a través de contratos temporales, que permiten una agilidad de cambio y unas posibilidades de modificación y elasticidad mayores.

- Una gran parte de las nuevas universidades creadas durante los últimos años, y también algunas de las más tradicionales, desarrollan una actividad claramente generalista (Michavila, Calvo, 1998). Con harta frecuencia persiguen, en esencia: sólo cubrir la tarea docente. De esa forma alcanzar una labor investigadora resulta difícil. La impartición de los ciclos formativos iniciales, básicos, de grado y licenciatura ocupa casi todos sus esfuerzos. Todo esto fue necesario, y sin duda útil, en un pasado no lejano, pero resulta del todo insuficiente cara al futuro (Miguel, 2000). Por el contrario, el doctorado no cuenta mucho. Apenas, con frecuencia, figura como un mero adorno, un complemento indispensable que aparece en la oferta curricular de los planes de estudio. Sus continuas reformas durante los últimos cursos constituyen una prueba palpable de querer transformar algo que no funciona de manera adecuada (Marcovitch, 2002). Pero dada la propia orientación, estructura y funcionamiento de la mayor parte de los centros universitarios resulta harto difícil proceder a cambios profundos en ello.

- En referencia con lo anterior, la organización básica de muchas Universidades españolas no está centrada en la tarea investigadora. De sobra es sabido que en un mundo tan desarrollado, científica y técnicamente, 
no es un asunto que se improvisa ni se pone en funcionamiento de manera sencilla. Sólo mediante una estrategia, a medio y largo plazo, es posible emprender ese camino, buscando la constante sinergia entre los diferentes entes, públicos y privados, que pueden contribuir a ello (Neave, 1984). Pero, lo grave en el caso español radica en que merced a su propia estructura tan homogénea, uniforme y con orientación docente generalista de la mayor parte de sus universidades, no resulta nada sencillo iniciar ese proceso de situar la investigación como objetivo central del quehacer universitario (Martínez López-Múñoz, 2001). Las limitaciones de todo tipo, materiales, formales y estructurales, son evidentes. Las primeras, acaso es más fácil superarlas, no así las otras.

Todas esas últimas deficiencias señaladas son comunes a las Universidades estatales y privadas. Si bien las razones que propician ese balance final es diferente. Numerosos resultados y análisis llegan a esa conclusión (Pérez Díaz, 2002). Aunque son distintas las causas que lo motivan y también son dispares los procedimientos a realizar cara a su corrección.

Lo antes referido es un asunto muy complejo, no procede pues realizar simplificaciones. Tampoco se dispone aquí del espacio suficiente para analizarlo con la profundidad que requiere. Voy a fijarme, en esencia y de manera algo más amplia, sólo en una cuestión: la posible repercusión territorial que implica cualquier proceso profundo de reestructuración del sistema universitario español. Pero éste, se refiere sólo a las Universidades que conforman el subsistema estatal, pues las Universidades privadas, dado que se mueven de manera más directa por los imperativos del mercado, y por ahora, siguen incrementando su número de alumnos de forma casi general, no parece que, a corto plazo, tengan razones tan urgentes para emprender un cambio de la dimensión y naturaleza espacial de su oferta.

Así, dada la preeminencia destacada de los centros estatales dentro del sistema universitario español, su análisis aporta una visión bastante ajustada de la realidad nacional, frente a la menor significación presente de las Universidades privadas. Las tres hipótesis centrales que se presentan, y que constituyen el centro básico de mi argumentación, son las siguientes:

i) Parece muy difícil mantener el actual sistema de centros con sus características y rasgos actuales durante los próximos años. La relación entre los costes económicos que origina su mantenimiento y los resultados prácticos obtenidos (docentes y de investigación) hace difícil sostener este sistema actual. Al paso de los años se advierten con mayor nitidez los profundos defectos estructurales que encierra. Cada vez son más arduos de ocultar o disimular dentro de un escenario que se abre sin cesar en su triple vertiente: nacional, europea comunitaria e internacional.

ii) Todo sugiere propiciar profundas reformas oportunas orientadas hacia la consecución de objetivos claros e irrenunciables. Éstos deben estar 
marcados por la siguiente finalidad: especialización de las diferentes universidades en su doble tarea básica, docente e investigadora. A la vez, se precisa establecer una jerarquización de las universidades. Por supuesto, ello debe llevarse a cabo de la forma más objetiva posible, renunciando a una homogeneidad, de hecho, más aparente que real. Ese ranking de centros tendría como objetivo impulsar la propia competitividad de todas las Universidades. De esa forma sería más factible proceder a la apertura de los centros hacia el exterior, dentro de las dispares escalas territoriales antes apuntadas.

iii) Nada aconseja ampliar la red de centros existente, menos aún si se mantienen los parámetros dominantes en los años últimos. Por el contrario, los esfuerzos mayores deben orientarse hacia la reestructuración de la mayoría de los existentes. Lo cual, por fuerza, implicará la desaparición de algunas especialidades, el reacomodo de otras, y la readaptación de unas terceras. Otra cosa bien diferente es que, a nadie se oculta, que tales cuestiones, si bien se enuncian con suma facilidad, su puesta en práctica es difícil, precisa valentía política y visión a largo plazo (Vázquez, 2007).

Dentro de un esfuerzo por sintetizar en extremo esta cuestión, estimo que existen dos tipos de problemas, esenciales y de diferente naturaleza: Los referentes a los recursos humanos y los de índole espacial y territorial.

Los primeros, de honda significación, por su propia singularidad, se apartan más del objetivo central de nuestra disciplina geográfica. Ello aconseja dedicarle menor atención. Sólo cabe recordar que el sistema universitario español, al igual que sucede con una buena parte del europeo, se sustenta, como es de sobra conocido y se ha señalado ya, sobre la estructura funcionarial: los puestos de trabajo una vez consolidados se prolongan toda la vida laboral. Los derechos adquiridos por estos empleados públicos se convierten en una considerable limitación a la hora de llevar a cabo reformas y cambios sustanciales. Limitan considerablemente la agilidad y la rapidez necesaria en esos procesos de cambio. De la misma manera, tampoco es fácil introducir mecanismos de estímulo que impulsen modificaciones por parte de los interesados. Las Universidades privadas al utilizar procedimientos relacionados con su personal, más acordes con la regulación laboral común, tienen problemas de otra naturaleza.

Por su parte, todo lo relacionado entre la universidad y el territorio constituye un extenso y complejo campo que permite y precisa diferentes orientaciones de análisis (Querrien, Lassave, 1994). Así, aparte de sus implicaciones estratégicas de más largo alcance, para toda ciudad donde se ubica un centro universitario conlleva una serie importante de ventajas, directas e indirectas. Sólo dentro de una perspectiva económica se considera que en ciudades universitarias de tamaño pequeño o medio, la Universidad se convierte en un destacado elemento de su vida productiva (Zerlang, 1997). Incluso en numerosas ciudades donde la puesta en funcionamiento de su Universidad es reciente, los beneficios 
y las expectativas que la acompañan hacen de ella una institución muy apreciada y de la que se espera obtener significativos beneficios. Para algunos es un claro motor de desarrollo económico (Egea et al., 2001).

Desde una perspectiva intraurbana, la dimensión e importancia de las transformaciones realizadas en España ha sido muy destacada. De un lado, la creación ex novo de decenas de campus universitarios han sido elementos fundamentales en los planes generales de expansión y ordenación urbana; convirtiéndose en nodos de clara atracción suburbana y periurbana (Campos Calvo-Sotelo, 1999). De otro, su impacto intraurbano no ha sido menor. Cientos de edificios históricos han sido remodelados y reacondicionados utilizándose en tareas relacionadas con la Universidad, en línea con lo sucedido en otros países de nuestro entorno (Feldestein, 1996). Se ha cumplido de forma sobrada el efecto impulsor tradicional de la universidad sobre la ciudad (Bender, 1988). De ahí el celo y el cuidado con que las autoridades municipales vigilan cualquier modificación que pueda afectar a "su Universidad".

Se entiende que, a medio y largo plazo, para cualquier territorio, organizado de forma política en región autónoma o no, contar con la dotación de una Universidad siempre es un activo destacado. En todo proceso de organización territorial las Universidades desempeñan un papel sobresaliente (SaintJulien, 1990-91). Más aún, resulta fácil valorar su rol significativo en todo proceso de desarrollo en nuestra sociedad actual (Florast, 1992). Esto explica y justifica que las consideraciones territoriales y estratégicas anhelan contar con estos servicios, y cuando se disfrutan, se pretende siempre incrementarlos. $\mathrm{Y}$ viceversa, se intenta detener y reducir cualquier remodelación que reduzca o aminore algo los equipamientos existentes. Se comprende que, dentro de una escala local, urbana o intraurbana, las preocupaciones por lo inmediato sean prioritarias. Son muchos los aspectos favorables y las consecuencias positivas derivadas del funcionamiento de una Universidad para la ciudad y su entorno inmediato (Genestier, 1996), como antes se indicó. En el caso español la expansión de centros universitarios durante las últimas décadas ha sido un elemento que ha contribuido a su proceso de terciarización (Serrano, 2000). A veces, estas cuestiones se enfocan como un asunto de prestigio local, real o ficticio. Aunque, a menudo, pesan mucho los propios intereses profesionales y personales; no se olvide que éstos desempeñan un papel destacado en muchas universidades públicas en España, dada su naturaleza y organización, donde la movilidad espacial de su personal, estudiantes y profesores, es reducida. Por el contrario, la endogamia lo preside todo. Ello implica una actitud de gran recelo hacia los cambios que acontecen en su equipamiento universitario. De ahí que en un futuro proceso de reorganización del sistema universitario en España, que implique reacomodos y cambios espaciales, cabe esperar la oposición rotunda por parte de los gobiernos pertinentes. Resulta paradógico que en el presente, cuando el sistema de Universidades públicas en España se encuentra saturado, a menudo se presentan incluso nuevas demandas de más centros por parte de los gobiernos de numerosas ciudades. 
Ahora bien, por encima de esa dimensión de análisis de escala local, urbana, el aspecto más preocupante, cara a una posible reorganización territorial o estructural del sistema de centros universitarios, descansa en el ámbito regional. En este sentido interesa hacer una aclaración destacada. A partir, y con el fundamento legal que aporta la Constitución de 1978, España inicia un proceso acusado y continuo de descentralización política administrativa y de construcción regional (Serrano, 1994 b). Las competencias asumidas por algunas regiones sobrepasan a las que son habituales en numerosos Estados de organización federal. Así, pues, toda la organización de los servicios públicos, y equipamientos terciarios es preciso hacerlo en esa clave territorial regional. Los conflictos de poderes que surgen entre el Estado Central y los gobiernos autonómicos son numerosos y continuos. La propia tarea armonizadora y de coordinación del gobierno nacional de España encuentra serias dificultades para contentar las demandas regionales que siempre, a pesar de su diferente significación demográfica y territorial interna, buscan un protagonismo y una significación homogénea. El efecto demostración, "lograr tanto como la que más", es una constante. Una espiral que se autoalimenta

Por consiguiente, un problema fundamental existente radica en la difícil armonía entre los subsistemas de centros universitarios regionales y el del conjunto español. Dada la pluralidad de intereses existente y la distribución efectiva de poderes autonómicos y centrales, no es fácil conseguir un resultado final equilibrado y aceptable por todos. Pero la realidad es tozuda e implacable. Al paso de los años se advierten las fuertes deficiencias que adolecen el sistema universitario español. Los problemas son tan graves que no pueden ocultarse. Existe un claro desequilibrio de coste/eficiencia y de demanda/oferta. Ello se manifiesta de forma más acusada en la dispar naturaleza de los centros politécnicos y los restantes. Los resultados finales son mejorables en grado extremo. Se impone una necesaria competitividad de las Universidades, en un mercado mundial más abierto. No es fácil poner en duda tales hechos. Tampoco ignorarlos por más tiempo.

Igualmente, es palpable que la reducción del número de estudiantes propicia una ocasión favorable, la cual debe aprovecharse para llevar a cabo profundas reformas estructurales en el sistema universitario. No tiene sentido mantener decenas de centros ofreciendo cientos de titulaciones, que casi se repiten de manera clónica y mimética. A la vez, en la mayoría de estos cursos, apenas se cubre un ligero porcentaje de las plazas disponibles; a menudo menos de un tercio. Mientras que, al mismo tiempo, se detectan numerosas y manifiestas carencias, precisamente en campos científicos de gran demanda y proyección estratégica, en especial los de naturaleza técnica. A título de ejemplo, refiero el caso de nuestra disciplina, la Geografía. No parece lógico que exista más de una veintena de universidades donde se ofrece la especialidad y el propio doctorado, cuando el número total de estudiantes inscritos en varias de ellas no suma más de unas escasas decenas de alumnos. Sería preferible concentrar los esfuerzos en seis o siete centros, haciendo de ellos lugares de mayor calidad y excelencia; 
también resultaría más barato su mantenimiento, incluso incluyendo el coste de los traslados de los alumnos a tales centros seleccionados. Su funcionamiento cotidiano conlleva, pues, además de numerosas consecuencias negativas, un derroche de medios palpable. Esas mismas sumas de dinero, dedicadas a otras inversiones, propiciarían resultados más satisfactorios. Es necesario construir un nuevo sistema universitario español moderno, abierto y competitivo. Las autoridades pertinentes de la Unión Europea así lo aconsejan.

Cuanto más se tarde en aplicar dichas reformas estructurales, más difícil será llevarlas a cabo y peor será la situación a la que se puede llegar. Pero, como se ha apuntado ya en varias ocasiones, se trata de una acción de alto contenido político. Los plurales sujetos afectados, con poderes directos en las diversas escalas territoriales, complican en extremo el camino a seguir. Es necesario situarse dentro de una visión a largo plazo. De poco sirven actitudes basadas en conservar a ultranza lo que se tiene. Eso sólo ayuda a descomponer más la realidad existente. En el mejor de los casos se retrasarán unas decisiones. No se pueden mantener instituciones aisladas de las grandes corrientes innovadoras mundiales. No sé hasta qué punto, el actual proceso de reforma universitaria, auspiciada por la Unión Europea, ayudará a transformar la Universidad Española. Sería deseable. No obstante, acaso, la inercia de los hechos continuará.

\section{BIBLIOGRAFÍA}

Almarcha Barbado A (2001) Misión de la Universidad. Enseñanza superior y competitividad: la globalización de los mercados. Revista Española de Investigaciones Sociológicas, REIS, 93: 205-219.

Álvarez Morales A (1972) Génesis de la Universidad Española Contemporánea. Instituto de Estudios Administrativos, Madrid.

Bender T (1988) The University and the City. From Medieval origins to the Present. Oxford University Press, Oxford.

Cabrera Montoya B, Báezdelafé B (2004) Reforma universitaria en España: La síntesis imposible entre la tradición y la mundialización. Sistema. Revista de Ciencias Sociales, 18: 33-54.

Campos Calvo-Sotelo P (1999) Las universidades en España: Historia, Urbanismo y Arquitectura. Secretaría General Técnica del Ministerio de Fomento, Centro de Publicaciones, Madrid.

Castells M (2005) The Network Society. Edward Elgar Publishing, Cheltenham.

Egea Día N, Mula Gómez A J, Torroba Ochoa P (2001) Universidad, autonomía y financiación. Universidad de Murcia, Murcia.

Embid Irujo A, Michavila Pitarch F (2001) Hacia una nueva Universidad. Apuntes para un debate. Tecnos, Madrid.

EUROSTAT (2006) Europe en Chiffres. Annuaire Eurostat, 2005. Commission Européenne, Luxembourg.

Felsestein D (1996) The university in the metropolitan area: impacts and public policy implications. Urban Studies, 33: 1556-1580. 
Florast R (1992) The University: a regional boost? Abebury, Aldershot.

Genestier P (1995) L'Université et la cité. Espaces et Societés, 30: 80-98.

Guereña J L, Fell E M, Aymes J M (eds.) (1991) L'Université en Espagne et en Amérique Latine du Moyen Age à nos jours. Publications de l'Université de Tours, Tours.

Grosetti M, Losego Ph (1996) La territorialisation de l'enseignement supérieur et de la recherche: France, Espagne et Portugal. CERS, París.

Hernández F (1990) Estructura ocupacional y mercado de trabajo en España. In Giner S (ed.) España, sociedad y política, Espasa Calpe, Madrid: 143-167.

Illeris S (1996) The services economy. A geographical approach. John Wiley \& Sons, Chichester, Nueva York.

Kagan R L (1981) Universidad y Sociedad en la España Moderna. Tecnos, Madrid.

Lamo de Espinosa E (2001) La reforma de la Universidad en la sociedad del conocimiento. Revista Española de Investigaciones Sociológicas, REIS, 93: 243-255.

Laszlo P (1996) Les universités américaines. Flammarion, París.

López Piñero J M (dir.) (1991) España, ciencia. Espasa-Calpe, Madrid.

Marcovitch J (2002) La Universidad (im)posible. Cambridge University Press, Madrid.

Martínez López-Muñoz J L (2001) Universidad y enseñanza superior. In Fundación para el Análisis de los Estudios Sociales (ed) El sistema educativo en la España de los 2000, Madrid: 111-185.

Mattelart A (2005) La sociedad de la información. Claves para comprender el nuevo orden internacional. In Jarauta F (ed) Foro de la mundialización. Agenda de la globalización. Problemas, CajaMurcia, Murcia: 49-74.

Michavila F, Calvo B (1998) La universidad española hoy. Editorial Sistema, Madrid.

Miguel A (1973) Diagnóstico de la Universidad. Guadarrama, Madrid.

Miguel J M de (2000) Reinventando la Universidad. Gestión y Análisis de Políticas Públicas, 17-18: 119-159.

Montoro Romero R (1981) La Universidad en la España de Franco (1939-1970). Un análisis sociológico. Centro de Investigaciones Sociológicas, Madrid.

Neave G (1984) On the road to Silicon Valley? The changing relationship betweenn Higher Education and Government in Western Europe. European Journal of Higher Education, 19(2): 34-51.

OECD (1998) Redefinig tertiary education. París.

Peach M (2001) Globalization of education in Spain: from isolation to internationalization to globalization. Higher Education in Europe, XXVI(1): 69-76.

Pérez Díaz V (2002) La crisis endémica de la universidad española. Claves de Razón Práctica, 158: $38-43$.

Querrien A, Lassave P (coord.) (1994) Universités et territoires. Les Annales de la Recherche Urbaine, 62-63.

Romero J (2006) La España inacabada. Universidad de Valencia, Valencia.

Saint-Julien T (1990-1991) L'Université et l'aménagement du territoire. L'Espace Géographique, XIX-XX(3): 206-210.

Salmon K (1995) The Modern Spanish Economy. Transformation and integration into Europe. Pinter, Londres. 
Serrano Martínez J M.a (2006) Extranjeros en España: residentes e inmigrantes. Situación presente y perspectivas inmediatas. Anales de Historia Contemporánea, 22: 25-53.

Serrano Martínez J M. a (2000) Equipamiento universitario y terciarización de las ciudades españolas. In Morales Matos G. (ed.) Actas, Turismo y ciudad, A.G.E., Las Palmas de Gran Canaria: 248-258.

Serrano J M. , King R (1994) Urban systems and regional organization in Spain, Brighton, University of Sussex.

Serrano Martínez J M. ${ }^{a}$ (1994) The creations of regions in Spain and its consequences in urban systems and its hierarchy. Bevas, Aardrijkskundige Studies, 1/94: 31-45.

Vázquez J A (2007) La Universidad innovadora. España, 2006, un balance. Economistas, 25(11): 324-327.

VV. AA. (2007) Informe Mensual. La Caixa, Barcelona.

Wright A (1977) The Spanish Economy, 1956-1976. The MacMillan Press, Londres.

Zerlang M (1997) The university and the city. Geo-Journal, 1(3): 241-246. 